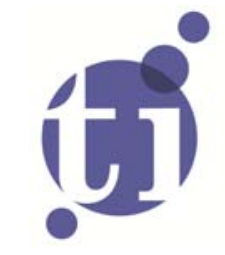

\title{
International Environmental Agreements for River Sharing Problems
}

Harold Houba

Gerard van der Laan

Yuyu Zeng 
Tinbergen Institute is the graduate school and research institute in economics of Erasmus University Rotterdam, the University of Amsterdam and VU University Amsterdam.

More TI discussion papers can be downloaded at http://www.tinbergen.nl

Tinbergen Institute has two locations:

Tinbergen Institute Amsterdam

Gustav Mahlerplein 117

1082 MS Amsterdam

The Netherlands

Tel.: +31(0)205251600

Tinbergen Institute Rotterdam

Burg. Oudlaan 50

3062 PA Rotterdam

The Netherlands

Tel.: +31(0)10 4088900

Fax: $+31(0) 104089031$

Duisenberg school of finance is a collaboration of the Dutch financial sector and universities, with the ambition to support innovative research and offer top quality academic education in core areas of finance.

DSF research papers can be downloaded at: http://www.dsf.nl/

Duisenberg school of finance

Gustav Mahlerplein 117

1082 MS Amsterdam

The Netherlands

Tel.: +31(0)20 5258579 


\title{
International Environmental Agreements for River Sharing Problems ${ }^{1}$
}

\author{
Harold Houba ${ }^{2} \quad$ Gerard van der Laan ${ }^{3} \quad$ Yuyu Zeng ${ }^{4}$
}

October 4, 2013

\footnotetext{
${ }^{1}$ This research is financially supported by Netherlands Organization for Scientific Research NWO grant 022.003.025. The authors thank Hans-Peter Weikard, Arantza Estévez-Fernández and Randolph Sloof and the participants of the seminar series Environmental Economics at WUR and Tinbergen Institute Mphil seminar for valuable suggestions. All derivations in Section 4.2 are given as an online appendix, https://sites.google.com/site/yves0805/research.

${ }^{2}$ H.E.D. Houba, Department of Econometrics and Tinbergen Institute, VU University Amsterdam, De Boelelaan 1105, 1081 HV Amsterdam, the Netherlands. E-mail: harold.houba@vu.nl

${ }^{3}$ G. van der Laan, Department of Econometrics and Tinbergen Institute, VU University Amsterdam, De Boelelaan 1105, 1081 HV Amsterdam, the Netherlands. E-mail: g.vander.laan@vu.nl

${ }^{4}$ Y. Zeng, Department of Econometrics and Tinbergen Institute, VU University Amsterdam, De Boelelaan 1105, 1081 HV Amsterdam, the Netherlands. E-mail: y.zeng@vu.nl
} 


\begin{abstract}
We study coalition formation and the strategic timing of membership of an IEA for environmental issues in the Coalitional Bargaining Game (CBG) of Gomes (2005, Econometrica). For the general CBG, we derive the necessary and sufficient condition for immediate formation of the grand coalition. We apply the CBG to a river sharing problem with two symmetric upstream agents and one downstream agent. Taking the discount factor and a productivity variable of water as parameters, we identify five regions in the parameter space. First, there is a region in which the grand coalition always forms immediately. Second, there are two regions in which there is for sure gradual coalition formation, in one of these regions there is a positive probability that the upstream agents form a monopoly. Third, there are two regions in which the grand coalition forms immediately with positive probability, but also gradual coalition formation might occur with positive probability.
\end{abstract}

Keywords: Coalitional Bargaining Game, International Environmental Agreements, River Sharing Problems, Markov Perfect Equilibrium, Efficiency, Monopoly

JEL codes: C78, Q25 


\section{Introduction}

In many trans-boundary environmental issues, such as international rivers and greenhouse gasses, countries engage in negotiations for international environmental agreements (IEAs) to deal with these issues. Although the economic benefits for reaching IEAs seem obvious, negotiations are often tedious, or fail altogether. Some countries are eager to participate, while other countries prefer delaying their participation or even abstain from it. Although strategic considerations are in principle suited for game theoretic analyses, the presence of externalities, the issue of timing participation and the huge number of possible coalitions in case of global IEAs makes application of game theory to explain IEAs a challenging task.

There are several strands of game theoretic approaches to study IEAs. In most of these studies, the partial-agreement Nash equilibrium of Chander and Tulkens (1995) determines the benefits from IEAs and it takes into account how other countries organize themselves in alternative IEAs. $I^{1}$ The cooperative approach has focused on transfer schemes that distribute the gains from forming the efficient grand coalition. These include the $\gamma$-Core as proposed in Chander and Tulkens (1995, 1997) and Germain et al. (2003); the extended Shapley value proposed in Macho-Stadler et al. (2007); and alternative axiomatic approaches in Ambec and Sprumont (2002), Ambec and Ehlers (2008), van den Brink et al.(2012). This approach has delivered many valuable insights about the impact of idealized principles on the distribution of the gains that arise from IEAs. Part of this literature also proposes non-cooperative procedures to implement cooperative solutions, but the underlying conditions needed to make these procedures work are often absent in reality. Another approach applies stylized strategic bargaining games to IEAs, e.g., Carraro et al. (2005a, 2005b) and Houba et al. (2000). Strategic bargaining games are very general, but their application is hampered by the difficulty of computing equilibria in empirical settings. Due to these difficulties, a third strand of literature has emerged that resorts to a hybrid way of modeling. It combines the strategic aspect of joining IEAs assuming cooperative sharing rules for distributing the IEAs' gains. In a cartel setting Eyckmans and Finus (2004) and Weikard (2009) propose a class of sharing rules that enhance stability. In particular, the last author establishes a condition for stability of the efficient grand coalition in environments with negative externalities. Comparison of the effects of these and other sharing rules on stability are investigated in laboratory experiments by McGinty et al. (2012) and in applied IEA climate models in e.g. Carraro et al. (2006), Weikard et al. (2006) and Nagashima et al. (2009).

In this paper we consider the application of the Coalitional Bargaining Game (CBG) proposed by Gomes (2005) to deal with strategic timing of participation and gradual coalition formation in negotiations for IEAs. The CBG is a dynamic game that is played during a number of rounds and in which the set of players and the stage game changes over time. The game starts with a set of players, but over time the players form coalitions, always at most

\footnotetext{
${ }^{1}$ These gains can be determined prior to the analysis and summarized as the partition function form. This form is taken as the primitive of many studies.
} 
one at a time. At the beginning of each round, a collection of coalitions is present, i.e., a partition of the players being the coalitions formed in the preceding rounds called a coalition structure. Then this coalition structure forms the updated set of players that bargaining among each other, so the set of players in each round is the set of coalitions in the coalition structure. The stage game of each round is as follows. One of these players (coalitions) in the coalition structure is randomly chosen and this player proposes a new coalition structure that is obtained from the present coalition structure by merging together two or more players (coalitions), including the proposing player, while all other players stay as they are. In addition, the proposing player (coalition) offers a payoff to every other player (coalition) in the proposed merger $2^{2}$ All other players of the newly proposed merger respond by either rejecting or accepting the offer. When they all accept, then the new coalition structure is formed and the proposing player pays the offers and keeps the remaining (anticipated) value of the coalition; when at least one player rejects, then nothing changes in the round under consideration. When the grand coalition is formed, then the game ends; otherwise it goes to the next round where the stage game restarts including the random selection of a new proposer.

The stage game of the CBG is a reduced form of a more realistic model with detailed contracts, see e.g. Gomes (2005). Although this more realistic model is prone to the mentioned difficulty of computing equilibria, this reference also demonstrates that important theoretical results for the realistic model can be easily derived from the CBG with the stage game in the reduced form. One result states that there always exists a Markov perfect equilibrium and that, in every coalition structure, every coalition has an endogenous reservation value. Another result states that when the grand coalition is Pareto efficient, then in each round the proposing coalition proposes to merge with at least one other coalition in the current coalition structure, and all members included in the newly proposed coalition approve. So, the set of coalitions in the coalition structure decreases with at least one in every round and the merging process continues until the grand coalition has formed, which takes at most as many rounds as the number of players at the start of the game minus one. Gomes (2005) gives a rather restrictive sufficient condition for the immediate emergence of the grand coalition, but this leaves the question what sequence of coalitions emerges otherwise. For IEAs, it is of interest whether the grand coalition immediately emerges or whether it forms through a sequence of mergers. This might explain why negotiations for some IEAs proceed fast, while other negotiations are tedious. For future negotiations, it might predict whether tedious negotiations with strategic timing can be expected.

The stage game in the CBG incorporates the strategic aspect of joining an IEA, an

\footnotetext{
${ }^{2}$ For technical simplicity, Gomes (2005) assumes that the proposing player buys the ownership rights of his proposed coalition. In order to buy out all other players in this coalition, it is implicit that the proposer is able to finance the amount needed. Alternatively, one might consider all players in a coalition as shareholders and the shares determine the payoffs. For the context of IEAs with streams of payoffs over time, the alternative interpretation is more appropriate but also it is more elaborate. Therefore, we will closely follow Gomes (2005).
} 
aspect that is prominent in the hybrid modeling approach, in the sense that one player has the initiative to ask others to join the IEA, who then decide whether to enter. One might argue that players who are not proposed to are excluded from becoming a member, but instead one could also argue that these players' reservation values are too expensive to join the IEA. Also, the proposer might deliberately let some players free ride on the proposed IEA. In the $\mathrm{CBG}$, every player has an endogenous reservation value depending upon the current coalition structure. These reservation values replace the exogenously given sharing rules in the hybrid models. The major novelty compared to these models is the modeling of a dynamic coalitional formation process in which agents are forward looking.

One aim of our analysis is to investigate gradual coalition formation and tractability of closed form solutions in the presence of an efficient grand coalition. Therefore we apply the CBG to a specific simple river sharing problem with three agents, in which one downstream agent is located at the junction of two symmetric tributaries, each belonging to a single upstream agent. All agents have decreasing returns to scale of the use of water. We normalize this situation such that we have two parameters: the discount factor and a productivity parameter, which describes the incremental value of a single upstream country merging with the coalition of the other two countries. Several very simple economic questions can be asked that do not have a straightforward answer without performing the equilibrium analysis. Should the two upstream agents first form a monopoly before jointly negotiating with the downstream agent? Or should each of them try to reach a bilateral agreement with the downstream agent first, trying to capture a share of the largest incremental value, or instead propose the grand coalition immediately? Similar for the downstream agent, should it contract with both supplying upstream agents at once, or first contract with one of them before expanding the coalition? This paper shows that the answers to these questions depend on the values of the discount factor and the productivity parameter. Besides analyzing the CBG associated to this specific simple river sharing problem, we also derive, for the general $\mathrm{CBG}$, a necessary and sufficient condition for the immediate emergence of the grand coalition, which extends a result in Gomes (2005).

The remainder of this paper is organized as follows. The river sharing problem and the general CBG are presented in Section 2. Section 3 analyzes Markov perfect equilibria in the general CBG and it includes the necessary and sufficient condition under which the grand coalition immediately forms. The equilibrium analysis for the river sharing problem is presented in Section 4 and Section 5 concludes.

\section{The Model}

In this section we introduce the specific simple river sharing problem and the general strategic $\mathrm{CBG}$ in two separate subsections. The general river sharing problem can be found in e.g. Houba et al. (2013) and the general CBG in Gomes (2005). For the purpose of this paper, 


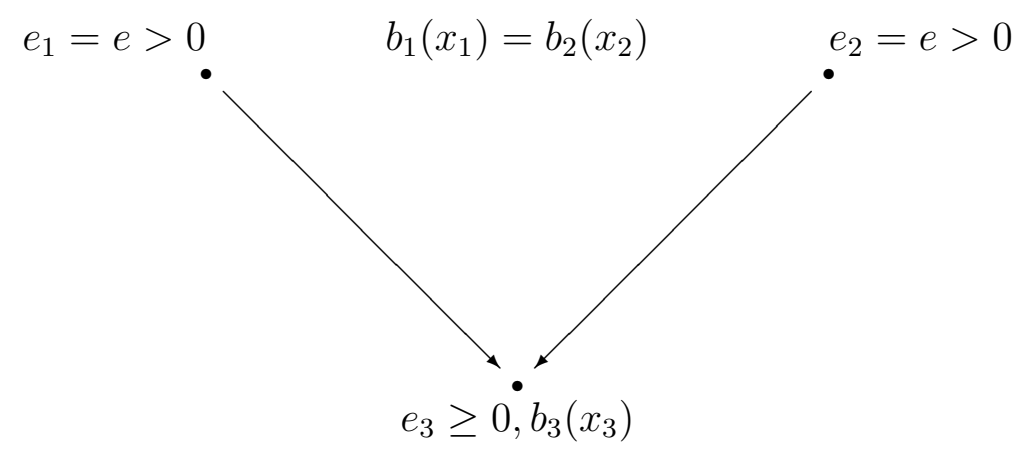

Figure 1: A V-shaped river with two merging tributaries.

it suffices to introduce a less general river sharing problem with three agents only.

\subsection{The River Sharing Problem}

We consider a river that flows through three locations that could be either irrigation plots, cities or countries. These are also called agents and $N=\{1,2,3\}$ denotes the set of agents. The river geography has two upstream agents, called 1 and 2, that represent two tributaries that merge at a common downstream location, called agent 3. Each agent $i, i \in N$, has its own local water resource $e_{i}$ with $e_{3} \geq 0$ and $e_{i}>0$ for $\left.i=1,2\right]^{3}$

Agent $i$ uses an amount of water $x_{i}$ that has to be feasible depending upon his access to water. Agent 1's feasible water use is given by $x_{1} \leq e_{1}$. Similarly, we have $x_{2} \leq e_{2}$ for agent 2. Available water for agent 3 consists of his own local water resource $e_{3}$ plus the inflows of water $e_{1}-x_{1}$ and $e_{2}-x_{2}$ received from his upstream neighbors. Feasible water use $x_{3}$ by agent 3 is bounded from above by this sum, which we write as $\sum_{i \in N} x_{i} \leq \sum_{i \in N} e_{i}$ for convenience. Agent $i \in N$ has a (net) benefit function $b_{i}\left(x_{i}\right)$ that is differentiable for all $x_{i}>0$ and strictly concave. Furthermore, there exists a satiation point $x_{i}^{S}>0$ such that $b_{i}^{\prime}\left(x_{i}^{S}\right)=0$. The benefits include the unmodeled costs of water extraction for each agent. In our analysis of the $\mathrm{CBG}$ for this river sharing problem, we treat both upstream locations symmetrically in terms of endowments $e_{1}=e_{2} \equiv e>0$ and benefit functions $b_{1}(z)=b_{2}(z)$, $z \in[0, e]$. This eases the computational burden and discussion. Figure 11 illustrates the river sharing problem analyzed in our study, which we will call the V-shaped river sharing problem.

We assume that the benefits are measured in terms of money and agents are able to transfer utility to each other by making monetary transfers. So, the benefits are transferable. The monetary transfer to agent $i$ is equal to $t_{i} \in \mathbb{R}$, where $t_{i}>0$ means that agent $i$ receives a monetary transfer from other agents and, otherwise, he pays in total a monetary transfer

\footnotetext{
${ }^{3}$ Here we do not allow $e_{i}=0$ for $i=1,2$. An upstream agent is irrelevant when it has zero water resources, because water flows from upstream to downstream. Furthermore, the water resource of agent 3 can only be utilized by agent 3, while the water of an upstream agent can be used by that agent and by the downstream agent 3 .
} 
$t_{i}<0$ to other agents. A monetary transfer scheme is a vector $t=\left(t_{1}, t_{2}, t_{3}\right)$ such that no financial deficit is allowed: $\sum_{i \in N} t_{i} \leq 0$. By the transferability assumption we have that each agent $i$ has a quasi-linear utility function $u_{i}\left(x_{i}, t_{i}\right)=b_{i}\left(x_{i}\right)+t_{i}$. We further take $x_{1}^{S} \geq e, x_{2}^{S} \geq e$, and $x_{3}^{S}>e_{3}$, i.e., for each agent its own inflow is at most equal to its satiation point with strict equality for agent three and assume that the marginal benefit of agent 3 at $e_{3}$ is higher than the marginal benefit of $i$ at $e_{i}, i=1,2$. By strict concavity of the benefit functions we have that $b_{1}$ and $b_{2}$ are increasing on $[0, e)$ and $b_{3}$ is increasing on $\left[0, e_{3}\right)$, while by the strict inequality $x_{3}^{S}>e_{3}$, agent 3 has an incentive to demand water from its upstream agents. Finally, we assume that all agents are utility maximizing and that all utility functions and water resources are common knowledge.

\subsection{The Coalitional Bargaining Game}

We now turn our attention to the general CBG of Gomes (2005) The set of players is denoted as $N=\{1,2, \ldots, n\}$. The coalition structure $\pi$ is a partition of $N$ describing who have formed coalitions already. The initial coalition structure is denoted by $\pi^{0}$ and it is the finest partition of $N$, i.e., $\pi^{0}=\{\{1\},\{2\}, \ldots,\{n\}\}$. The CBG is a dynamic game where, at every round, a player (being a coalition in $\pi$ ) is randomly chosen to propose an offer to a set of other players (coalitions) in the coalition structure, who can either accept or decline the offer. For ease of notation, we assume invariant probabilities in selecting who proposes throughout all rounds and all coalition structures. Formally, there is a probability distribution $p=\left(p_{i}\right)_{i \in N}$ with $p_{i}>0$ for all $i$, and at the beginning of a bargaining round $\tau$, the probability of a coalition $C$ is selected to be the proposer with probability $p_{C}=\sum_{k \in C} p_{k} 5^{56}$

The rules of the CBG are as follows. Let $\pi$ be the coalition structure at the start of some bargaining round $\tau$ and $S \subseteq \pi$ be a collection of coalitions in $\pi$. Then the coalitions in $S$ can bargain and form a new coalition $C^{\prime}=\cup_{B \in S} B$, changing the coalition structure to $\pi^{\prime}=\left\{C^{\prime}\right\} \cup(\pi \backslash S)$, where $C^{\prime}$ is the set of all (singleton) players in $N$ belonging to one of the coalitions in $S$. To be more precisely, at the start of a round a coalition $C$ in the coalition structure $\pi$ is randomly chosen with probability $p_{C}$ to be the proposer. This coalition then proposes the pair $(S, t)$, where $S \subseteq \pi$ with $C \in S$ is a collection of coalitions that are proposed to merge into $C^{\prime}$, and the vector $t=\left(t_{B}\right)_{B \in S \backslash\{C\}}$ consists of the proposed monetary offers $t_{B} \in R$ made by $C$ to each coalition $B \in S \backslash\{C\}$. The coalitions in the collection $S$ that are proposed to, i.e., the coalitions in $S \backslash\{C\}$, respond sequentially in a fixed order (the order of response turns out to be irrelevant), either accepting or rejecting the offer. If these coalitions are unanimous in accepting the offer, then the new coalition

\footnotetext{
${ }^{4}$ For a complete description of this game and the motivation of the underlying assumptions, we refer to this reference.

${ }^{5}$ Our general results also hold if we would allow for general probabilities $p_{C}(\pi)$ that depend on the coalition structure. Okada $(1996,2000,2011)$ also assumes invariant probabilities.

${ }^{6}$ In the specific simple river sharing problem with three agents, we simply assume $p_{1}=p_{2}=p_{3}=\frac{1}{3}$ and $p_{\{1,2\}}=p_{\{1,3\}}=p_{\{2,3\}}=\frac{2}{3}$.
} 
structure $\pi^{\prime}=\left\{C^{\prime}\right\} \cup(\pi \backslash S)$ immediately forms in round $\tau$ and the proposing coalition $C$ divides the continuation payoff of coalition $C^{\prime}$ in $\pi^{\prime}$ by paying every joining coalition $B \in S$ the proposed lump-sum transfer $t_{B}$ and keeping the remainder 7 If the responders are not unanimous in accepting the offer, then the next round's coalition structure equals this round's coalition structure, i.e., the coalition structure does not change, and there are no payments. We then write $\pi^{\prime}=\pi$. After accepting or rejecting the proposal the game ends when $\pi^{\prime}=\{N\}$, (which can only happen when the proposal $(\pi, t)$ was accepted.) Otherwise, the game proceeds into the next round where the stage game is played once again and a coalition from $\pi^{\prime}$ is chosen randomly to be the proposer. Notice that according to the rules that once a coalition has formed, it never breaks up ${ }^{8}$ and the renegotiation possibilities only allow for further merging of coalitions in the coalition structure. Furthermore it is assumed that all players have perfect information about the past play in the game when they make decisions.

Many studies take the payoffs associated with IEAs as a primitive of the model, where it is taken into account how other countries organize themselves in alternative IEAs. This is also the case in Gomes (2005) albeit that these payoffs represent the payoffs per round of infinite streams of these payoffs. In order to avoid confusion, we will refer to payoffs per round as disagreement values.

- disagreement value: $d_{C}(\pi)$ is the disagreement payoff for coalition $C$ in $\pi$, i.e., it is the value that coalition $C$ obtains per round when $\pi$ is the coalition structure.

Notice that even for $\pi=\{N\}$ we call $d_{N}(\{N\})$ the disagreement value for the grand coalition. Infinite streams of disagreement values are discounted by the common discount factor $\delta \in[0,1)$. Formally, we will work with normalized discounted payoffs $(1-\delta) \sum_{\tau=0}^{\infty} \delta^{\tau} x^{\tau}$, where $x^{\tau}$ denotes the payoff in round $\tau$. The merit of this normalization is that if we let $\delta$ go to 1 , then the normalized sum remains bounded.

Finally, following Gomes (2005), we will investigate mixed strategies and we assume that all players (coalitions) in this game have von Neumann-Morgenstern preferences.

\section{Markov Perfect Equilibria}

In this section, we will follow Gomes (2005) and study mixed Markov perfect equilibrium (MPE) of the general CBG. As is shown in this reference, mixed MPEs are necessary for the existence of MPEs and therefore unavoidable in any analysis. We first explain how to characterize mixed MPEs and then derive a necessary and sufficient condition for the immediate emergence of the grand coalition in an MPE. We do so in separate subsections.

\footnotetext{
${ }^{7}$ Recall Footnote 2. Gomes (2005) assumes that coalition $C$ becomes the sole owner of coalition $C^{\prime}$ after buying off all owners $B \in S \backslash\{C\}$. Alternatively, but more elaborate, we argued to consider the members of $C^{\prime}$ as shareholders whose share of current and future disagreement values is worth the offers $t_{B}$.

${ }^{8}$ Readers interested in a setting where coalitions may break up are referred to Gomes and Jehiel (2005).
} 


\subsection{Strategies and Equilibria}

Markov strategies assume that the $\tau$-th round strategy of a player depends only on the 'state' of the game at the start of round $\tau$. In the CBG, the state in round $\tau$ is the coalition structure at the start of round $\tau$. Thus, Markov strategies only depend on the coalition structure $\pi$. At the stage game of round $\tau$, the coalitions in the coalition structure make decisions: one coalition makes a proposal and the others who are proposed to respond. Therefore, following Gomes (2005), we assume that in every stage game the strategies of all players that belong to a same coalition $C$ coincide. Under this assumption we define for every coalition structure $\pi$ a stage-game strategy for every coalition $C \in \pi$ with the interpretation that the stagegame strategy of $C \in \pi$ is the stage-game strategy of every player $i \in C$, avoiding in this way duplication of strategies. Given a coalition structure $\pi$, the pure stage-game strategy of coalition $C \in \pi$ is composed of a proposal when selected as the proposer and a response when being proposed to by other coalitions. In a (behavioral) mixed stage-game strategy of coalition $C \in \pi$, this coalition possibly randomizes over the set of actions available when taking the decision. Thus, coalition $C$ randomizes over the set of all proposals $(S, t)$ and coalition $C$ randomizes over accept and reject when being proposed to.

Mixed Markov strategies induce present values of infinite streams of expected disagreement values, simply called values, for every coalition that depend on the coalition structure $\pi$. In addition, we refer to these values as the equilibrium values in a MPE. So, we have the following notion of value.

- $v_{C}(\pi)$ is the continuation (MPE) value for coalition $C$ in coalition structure $\pi$ at the beginning of round $\tau$ prior to the randomization that determines who becomes the proposing player in this round.

These values together with the stage-game Markov strategies have to be determined by the equilibrium analysis. Notice that the value for the grand coalition is trivial and equal to the infinite stream of disagreement values under coalition structure $\pi=\{N\}$, i.e.,

$$
v_{N}(\{N\})=(1-\delta) \sum_{\tau=0}^{\infty} \delta^{\tau} d_{N}(\{N\})=d_{N}(\{N\}) .
$$

So when the grand coalition is formed, the value of $N$ is equal to the disagreement value.

As shown in Gomes (2005) for any coalition structure, the response of any coalition in any MPE stage-game strategy is always a pure strategy in which the responder always accepts when indifferent. Formally, when a coalition $C \in \pi$ has to respond to a proposal $(S, t)$ made by another coalition $C^{\prime} \in \pi$, i.e., $C \in S \subset \pi$ and $t$ is a vector of offers including an offer $t_{C}$ of $C^{\prime}$ to $C$, then $C$ accepts if and only if $t_{C} \geq t_{C}(\pi)$, where $t_{C}(\pi)$ is the threshold value of $C$ in $\pi$ defined as

$$
t_{C}(\pi)=\delta v_{C}(\pi)+(1-\delta) d_{C}(\pi)
$$


When $C$ rejects the offer, it gets discounted continuation value plus the normalized disagreement value in this round. For future reference, we write $t(\pi)=\left(t_{B}(\pi)\right)_{B \in S \backslash\{C\}}$.

Now we consider the pure stage-game strategy of a coalition $C \in \pi$ when $C$ is chosen to be the proposer. When $C \in \pi$ proposes $(S, t)$, the responding coalitions in $S$ will all accept the proposal if and only if the offer to any $B \in S \backslash\{C\}$ is at least equal to its threshold value $t_{B}(\pi)$. It follows that $C$ extracts the highest surplus from proposing $S$ when offering exactly these threshold values of $t(\pi)$ to every $B \in S \backslash\{C\}$. When $C$ proposes $(S, t(\pi))$ knowing all responding coalition in $S$ accept, then coalition $C^{\prime}=\cup_{B \in S} B$ immediately forms, $\pi^{\prime}=\left\{C^{\prime}\right\} \cup(\pi \backslash S)$ immediately becomes the new coalition structure and the value to coalition $C$ is equal to

$$
v_{C}((S, t(\pi)) \mid \pi)=\delta v_{C^{\prime}}\left(\pi^{\prime}\right)+(1-\delta) d_{C^{\prime}}\left(\pi^{\prime}\right)-\sum_{B \in S \backslash\{C\}} t_{B}(\pi)
$$

This value is equal to the discounted continuation value $v_{C^{\prime}}\left(\pi^{\prime}\right)$ under the new coalition structure $\pi^{\prime}$ plus the normalized disagreement value under $\pi^{\prime}$ minus the sum of the equilibrium offers to the joining coalitions in $S$. The maximal attainable surplus that coalition $C$ can obtain from proposing any $(S, t(\pi))$ is defined as

$$
v_{C}^{*}(\pi)=\max _{S \subseteq \pi, S \ni C} v_{C}((S, t(\pi)) \mid \pi)
$$

So, when $C \in \pi$ is chosen to be the proposer and it plays a pure MPE stage-game strategy $(S, t)$, then the proposed $S$ of this MPE strategy must be a maximizer of (3) and the associated transfers $t=t(\pi)$ offer exactly the threshold value $t_{B}(\pi)$ to any $B \in S \backslash\{C\}$. Notice that we allow that coalition $C$ proposes $S=\{C\}$, i.e., it proposes to stay alone, guaranteeing itself $\delta v_{C}(\pi)+(1-\delta) d_{C}(\pi)$. Staying alone is the unique best response when $v_{C}\left(\left(S^{\prime}, t(\pi)\right) \mid \pi\right)<\delta v_{C}(\pi)+(1-\delta) d_{C}(\pi)$ for every $S^{\prime}$ containing some coalition $B \in \pi \backslash\{C\}$.

The existence result for MPE in Gomes (2005) is for mixed MPE in which a proposing coalition $C$ is allowed to randomize over several coalitions $S$ that are maximizers of (3) with the understanding that the MPE proposal is $(S, t(\pi))$. Let $\sigma_{C}(S \mid \pi) \geq 0$ be the probability that $C$ proposes $(S, t(\pi))$ with $\sum_{S \subset \pi, S \ni C} \sigma_{C}(S \mid \pi)=1$. Then a mixed Markov strategy for the proposal of $C$ in a stage game with coalition structure $\pi$ is optimal if and only if

$$
\sigma_{C}(S \mid \pi)=0 \text { if } v_{C}((S, t(\pi)) \mid \pi)<v_{C}^{*}(\pi) \text { for all } S \subseteq \pi, S \ni C .
$$

We end this section with the value of $C \in \pi$ in a mixed strategy MPE. When $C$ is the chosen to be the proposer it realizes payoff $v_{C}^{*}(\pi)=\max _{S \subseteq \pi, S \ni C} v_{C}((S, t(\pi)) \mid \pi)$ from playing an optimal mixed Markov strategy. This happens with probability $p_{C}$. When some other coalition $B \in \pi$ is the proposer and proposes $S$ including $C$, then $C$ gets it threshold value $t_{C}(\pi)$. This happens with probability $\sum_{B \in \pi \backslash\{C\}}\left[p_{B} \sum_{\substack{S \ni \bar{B}, C \\ \sigma_{B}}} \sigma_{B}(S \mid \pi)\right]$. Finally, when 
$B$ proposes a collection $S$ not including $C$, then the expected payoff of $C$ is given by $t_{C}\left(\pi^{\prime}\right)$, where $\pi^{\prime}$ is the coalition structure resulting from the proposal of $B$. This happens with probability $\sum_{B \in \pi \backslash\{C\}}\left[p_{B} \sum_{\substack{S \subseteq B \\ S \ni C \notin \Phi}} \sigma_{B}(S \mid \pi)\right]$. It follows that

$$
v_{C}(\pi)=p_{C} v_{C}^{*}(\pi)+t_{C}(\pi) \sum_{B \in \pi \backslash\{C\}}\left[p_{B} \sum_{\substack{S \subseteq \pi \\ S \ni B, C}} \sigma_{B}(S \mid \pi)\right]+t_{C}\left(\pi^{\prime}\right) \sum_{B \in \pi \backslash\{C\}}\left[p_{B} \sum_{\substack{S \subseteq \pi \\ S \ni B, C \notin S}} \sigma_{B}(S \mid \pi)\right] .
$$

Since $\sum_{S \subseteq \pi \mid S \ni B, C} \sigma_{B}(S \mid \pi)=1$ when $B=C$ it follows that $v_{C}(\pi)$ can be rewritten as

$$
\begin{aligned}
v_{C}(\pi) & =p_{C}\left(v_{C}^{*}(\pi)-t_{C}(\pi)\right) \\
& +t_{C}(\pi) \sum_{B \in \pi}\left[p_{B} \sum_{\substack{S \subseteq \pi \\
S \ni B, C}} \sigma_{B}(S \mid \pi)\right]+t_{C}\left(\pi^{\prime}\right) \sum_{B \in \pi \backslash\{C\}}\left[p_{B} \sum_{\substack{S \subseteq \pi \\
S \ni B, C \notin S}} \sigma_{B}(S \mid \pi)\right] .
\end{aligned}
$$

In the above expression, the value function is composed of two parts: the first part (the first term of the right-hand side) is the maximal net surplus from being the proposer if all coalitions $B \in S$ including proposing coalition $C$ are paid $t_{B}(\pi)$. This net surplus is the advantage to propose. Furthermore, for every merger of coalitions $S \subseteq \pi$ and all coalitions $B \in S$, the net surplus of proposing the coalition $S$ is the same. In MPE, each coalition in $\pi$ randomizes over those $S$ that maximize this coalition's net surplus, see Gomes (2005). The second part (the second and third term of the right-hand side) represents coalition $C$ 's status quo value. Note that the status quo value depends on other players' mixed strategies as well. For instance, the second term is the status quo value when $C$ is included in the proposal and the third term describes the situation when $C$ is not included in the proposal.

\subsection{Immediate Formation of the Grand Coalition}

In this section we first impose a sufficient condition that assures that in every bargaining round at least two coalitions in the coalition structure merge together, i.e., under the assumption every proposing coalition proposes to merge with at least one other coalition. It follows that under this assumption the grand coalition forms in at most $n-1$ rounds, because in a MPE a proposal is always accepted.

Assumption 3.1. For every coalition structure $\pi \neq\{N\}$ it holds that $\sum_{B \in \pi} d_{B}(\pi)<$ $d_{N}(\{N\})$.

Lemma 3.2 (Gomes, 2005). Under Assumption 3.1 it holds that for every $\pi \neq\{N\}$ and every $C \in \pi$ that $\sigma_{C}(\{C\} \mid \pi)=0$. 
Although Gomes (2005) provided a proof, we will give an alternative proof in the Appendix.

Assumption 3.1 means that only the grand coalition can fully internalize the externalities and therefore it follows that the maximum surplus from being the proposer (i.e., the first term of the right-hand of equation (5) ) is positive. Therefore in every coalition structure every proposer will propose to merge with at least one other coalition, thus $\sigma_{C}(\{C\} \mid \pi)=0$. Notice that, in any mixed MPE, the proposer may randomize over several coalitions and that he always makes proposals of the form $(S, t(\pi))$ that will be accepted. So, the proposer will reach some agreement for sure.

The consequence of this result is that each coalition $C \in \pi$ will make an acceptable proposal for sure and that the state moves from $\pi$ to $\pi^{\prime}$, where $S \neq\{C\}$ depends upon the coalition proposed by $C$. An intuitive explanation is that players can renegotiate the contract and the efficient grand coalition acts as an attractive sink of the Markov process to which the system converges. Similar results can be found in the literature (e.g., Chatterjee et al. (1993), Okada (2000)). The fact that the equilibrium path converges to the grand coalition is based on the assumptions that the coalition does not break up and the efficiency of the grand coalition.

We next state several results under the assumption that it is optimal to propose the grand coalition. The first lemma yields the continuation payoff of a coalition $C \in \pi$ when it is optimal for every $B \in \pi$ to propose $S=\pi$, thus to propose the grand coalition $N=$ $\cup_{D \in S} D=\cup_{D \in \pi} D$ with probability 1 .

Lemma 3.3 (Gomes, 2005). For a coalition structure $\pi \neq\{N\}$, let $\sigma_{B}(\pi \mid \pi)=1$ for every $B \in \pi$. Then it holds that

$$
v_{C}(\pi)=d_{C}(\pi)+p_{C}\left(d_{N}(\{N\})-\sum_{B \in \pi} d_{B}(\pi)\right), \text { for every } C \in \pi .
$$

In Gomes (2005), this result is stated without proof and we provide a proof in the Appendix.

The lemma states that the value for a coalition $C \in \pi$ when every coalition in $\pi$ proposes to form the grand coalition is equal to its disagreement payoff $d_{C}(\pi)$ under coalition structure $\pi$ plus a fraction $p_{C}$ of the net surplus $\left(d_{N}(\{N\})-\sum_{B \in \pi} d_{B}(\pi)\right)$. The probability to propose can be regarded as a measure of bargaining power and be taken as a coalition's bargaining weight in the asymmetric Nash bargaining solution.

In order to state the next result, we need some additional notation. For two coalition structures $\pi$ and $\pi^{\prime}$, we denote $\pi^{\prime} \leq_{p} \pi$ if $\pi^{\prime} \neq \pi$ can be obtained from $\pi$ by merging coalitions together, i.e., $\pi^{\prime}$ is coarser than $\pi 9^{9}$ The next theorem states that under Assumption 3.1 it is optimal for every proposer in a coalition structure $\pi \neq\{N\}$ and every coarser coalition structure $\pi^{\prime} \leq_{p} \pi$ to propose the grand coalition if and only if the discount factor is at

\footnotetext{
${ }^{9}$ Formally, we denote $\pi^{\prime} \leq_{p} \pi$ if $\left|\pi^{\prime}\right| \leq|\pi|$ and for every $B \in \pi^{\prime}$ there exist $S \subseteq \pi$ such that $B=S^{\cup}$. So $\pi^{\prime} \leq_{p} \pi$ and $\pi \leq_{p} \pi^{\prime}$ if and only if $\pi=\pi^{\prime}$. Moreover, any $\pi^{\prime} \leq_{p} \pi$ such that $\pi^{\prime} \neq \pi$ is obtained from $\pi$ after merging coalitions together. Notice that $\pi^{\prime}=\{N\}$ if and only if $\left|\pi^{\prime}\right|=1$.
} 
most equal to some upper bound. Let $S \subset \pi$ be a proper collection of subsets of $\pi$, thus $\cup_{B \in S} B \neq N$, and suppose that a member of $S$ proposes to form $\cup_{B \in S} B$. Let $\pi_{S}$ be the new coalition structure, thus $\pi_{S}=\left\{S^{\cup}\right\} \cup(\pi \backslash S)$, where $S^{\cup}$ denotes $\cup_{B \in S} B{ }^{10}$ Now, define

$$
\delta_{\pi}(S)=\frac{d_{N}(\{N\})-d_{S} \cup\left(\pi_{S}\right)-\sum_{B \in \pi \backslash S} d_{B}(\pi)}{\left(d_{N}(\{N\})-\sum_{B \in \pi} d_{B}(\pi)\right) \sum_{B \in \pi \backslash S} p_{B}+p_{S^{\cup}}\left(d_{N}(\{N\})-\sum_{B \in \pi_{S}} d_{B}\left(\pi_{S}\right)\right)}
$$

and

$$
\delta(\pi)=\min _{S^{\cup} \neq N} \delta_{\pi}(S)
$$

Finally we define

$$
\bar{\delta}(\pi)=\min _{\left\{\pi^{\prime} \leq{ }_{p} \mid \pi^{\prime} \neq\{N\}\right\}} \delta\left(\pi^{\prime}\right) .
$$

We show the following result.

Theorem 3.4. Let $\pi \neq\{N\}$. Under Assumption 3.1, for every proposer in the subgame at state $\pi$ and every proposer in every subgame at state $\pi^{\prime} \leq_{p} \pi$ it is optimal to propose $S=\pi$ if and only if $\delta \leq \bar{\delta}(\pi)$.

The MPE strategies underlying this result are pure strategies. Intuitively, if $\delta$ is large enough, delay of forming the grand coalition might occur because then the efficiency loss due to the delay of the grand coalition formation is relatively small. In such a case proposers may have an incentive to search for a better bargaining position, e.g., propose a partial coalition instead of the grand coalition. ${ }^{11}$ When $\delta \leq \bar{\delta}(\pi)$, then the grand coalition will be proposed immediately in $\pi$ and also in any coalition structure $\pi^{\prime} \leq_{p} \pi$, so in any coalition structure $\pi^{\prime} \leq_{p} \pi$ that might evolve in the sequence of bargaining rounds that will occur when a coalition $C \in \pi$ deviates from the equilibrium path and the grand coalition does not form immediately from $\pi$. Notice that, by definition, $\bar{\delta}(\pi) \leq \delta(\pi)$ and for $\delta>\bar{\delta}(\pi)$ we distinguish $\bar{\delta}(\pi)=\delta(\pi)$ from $\bar{\delta}(\pi)<\delta(\pi)$. First, in case $\bar{\delta}(\pi)=\delta(\pi)$, i.e., the minimum over $\pi^{\prime} \leq_{p} \pi$ is attained at the current coalition structure $\pi$, then proposing the grand coalition is clearly not optimal in coalition structure $\pi$ because of $\delta>\bar{\delta}(\pi)=\delta(\pi){ }_{12}^{12}$ Second, if $\bar{\delta}(\pi)<\delta(\pi)$, i.e., the minimum is attained for some coarser $\pi^{\prime} \neq \pi$, then for $\bar{\delta}(\pi)<\delta<\delta(\pi)$, there is some $\pi^{\prime} \leq_{p} \pi, \pi^{\prime} \neq \pi$ at which for at least one coalition in $\pi^{\prime}$ it is not optimal to propose the grand coalition. As a result it might be the case that, on the equilibrium path, for some coalition in $\pi$ it is better to make a proposal leading to $\pi^{\prime}$ (in one or more bargaining rounds)

\footnotetext{
${ }^{10}$ When $C \in S$ is the proposer, we denoted above $S^{\cup}$ by $C^{\prime}$ and $\pi_{S}$ by $\pi^{\prime}=\left\{C^{\prime} \cup(\pi \backslash S)\right\}$.

${ }^{11}$ In Section 4.2, we will identify a subclass of parameter values in the $\mathrm{V}$-shaped river sharing problem in which the upstream agents form a coalition acting as a monopolist to extract more surplus in the future negotiation round.

${ }^{12}$ This is the case for the initial coalition structure of the V-shaped river sharing problem analyzed in Section 4.2.
} 
instead of moving to the grand coalition immediately. However, in this situation it might also be the case that, on the equilibrium path, for every coalition in $\pi$ it is still optimal to move to $N$ immediately, only when a coalition deviates and the off-the-equilibrium-path coalition structure $\pi^{\prime}$ is reached it is not optimal anymore to form $N$, which in equilibrium cannot occur.

Theorem 3.4 implies that the grand coalition is formed immediately from the initial coalition structure $\pi^{0}$ consisting of single players when $\delta \leq \bar{\delta}\left(\pi^{0}\right)$. If this holds then it is also optimal to propose the grand coalition immediately for every $\pi \neq\{N\}$. Furthermore, let $\pi$ be a coalition structure with $|\pi|=2$, so $\pi$ consists of two coalitions, say $C_{1}$ and $C_{2}$. Then

$$
\bar{\delta}(\pi)=\min _{\left\{\pi^{\prime} \leq \pi:\left|\pi^{\prime}\right| \neq 1\right\}} \delta\left(\pi^{\prime}\right)=\delta(\pi)=\min _{S=\left\{C_{1}\right\}, S=\left\{C_{2}\right\}} \delta_{\pi}(S)=1
$$

because taking either $S=\left\{C_{1}\right\}$ or $S=\left\{C_{2}\right\}$ yields $\delta_{\pi}(S)=1$. So, this implies that from a coalition structure with two coalitions the grand coalition is formed immediately for every value of $\delta \in[0,1)$ and, thus, Lemma 3.2 is a special case of Theorem 3.4. This is in accordance with the results for the bilateral alternating-offer model with random proposers in e.g. Muthoo (1999), Moreover, every coalition structure $\pi$ always contains coarser coalition structures $\pi^{\prime}$ with two coalitions and, hence, $\bar{\delta}(\pi) \leq 1$.

Finally, we investigate the necessary and sufficient condition for the grand coalition to form immediately in all $\pi^{\prime} \leq_{p} \pi^{0}$ for all $\delta \in[0,1)$, i.e., $\bar{\delta}\left(\pi^{0}\right)=1$. Notice that $\bar{\delta}\left(\pi^{0}\right)=1$ if and only if $\delta_{\pi}(S) \geq 1$ for every $\pi \neq\{N\}$ and every $S \subset \pi$. So, $\bar{\delta}\left(\pi^{0}\right)=1$ if and only if for every $\pi \neq\{N\}$ and every $S \subset \pi$ it holds that

$$
d_{N}(\{N\})-d_{S} \cup\left(\pi_{S}\right)-\sum_{B \in \pi \backslash S} d_{B}(\pi) \geq\left(d_{N}(\{N\})-\sum_{B \in \pi} d_{B}(\pi)\right) \sum_{B \in \pi \backslash S} p_{B}+p_{S} \cup\left(d_{N}(\{N\})-\sum_{B \in \pi_{S}} d_{B}\left(\pi_{S}\right)\right)
$$

By rearranging terms this reduces to the inequality

$$
d_{S \cup}\left(\pi_{S}\right)+p_{S} \cup\left(d_{N}(\{N\})-\sum_{B \in \pi_{S}} d_{B}\left(\pi_{S}\right)\right) \leq \sum_{B \in S}\left[d_{B}(\pi)+p_{B}\left(d_{N}(\{N\})-\sum_{B \in \pi} d_{B}(\pi)\right)\right]
$$

From above, we know that these inequalities only impose restrictions for coalition structures with three or more coalitions, i.e., $|\pi| \geq 3$. This yields the following corollary.

Corollary 3.5. For every subgame at state $\pi$ and every proposer in $\pi$ it is optimal to propose the grand coalition if and only if for every $\pi$ such that $|\pi| \geq 3$ and every $S \subset \pi$ inequality (7) holds.

Proposition 4 of Gomes (2005) states that the grand coalition is formed immediately if the inequality (7) holds, i.e., is a sufficient condition. The corollary shows that Proposition 4 
follows as a special case from Theorem 3.4 and, moreover, that it is a necessary and sufficient condition. Moreover, we also conclude that coalition structures with two coalitions can be neglected.

\section{MPE in the River Sharing Problem}

In this section we apply the CBG to the specific simple river sharing problem introduced in Section 2.1. Before we do so, we first have to derive the disagreement values of Section 3.2 and then obtain a suitable normalization to reduce the computational burden of the equilibrium analysis.

\subsection{Disagreement Values}

Payoffs in the CBG consist of infinite streams of disagreement values and all disagreement values are derived from the partial-agreement Nash equilibrium of Chander and Tulkens (1995) for each coalition structure in the economic model under consideration, which is the V-shaped river sharing problem of Section 2.1 that is summarized in Figure 1 .

Given $N=\{1,2,3\}$, there are five coalition structures to consider. Recall that the initial situation in which no coalition has formed is denoted by $\pi^{0}$, so $\pi^{0}=\{\{1\},\{2\},\{3\}\}$. For $i \in N=\{1,2,3\}$, we further denote by $\pi^{i}=\{\{i\}, N \backslash\{i\}\}$ the situation in which agent $i$ is single and the other two agents have formed a pair. Finally, the coalition structure in which the grand coalition has formed is denoted as $\pi^{N}=\{N\}$.

In the $\mathrm{V}$-shaped river sharing problem, optimal cooperation between the unconnected upstream agent 1 and 2 boils down to doing what each of these agents would do as a single agent. Optimal cooperation between agents 1 (2) and 3 never causes externalities on agent 2 (1). The absence of externalities implies that the disagreement value $d_{i}(\pi)$ of a single agent $i$ is independent of $\pi$, and we therefore denote $d_{i}=d_{i}\left(\pi^{0}\right)=d_{i}\left(\pi^{i}\right)$. Notice that a two agent coalition $\{j, k\}$ only appears in $\pi^{i}, i \neq j, k$, and so we denote $d_{j k}=d_{\{j, k\}}\left(\pi^{i}\right)$. We also denote $d_{N}=d_{N}\left(\pi^{N}\right)$. As a consequence of the quasi-linear utility, the maximal utility within each coalition is obtained by utilitarian welfare maximization of their joint benefits under the water availability constraints, see e.g. Houba et al. (2013). So,

$$
\begin{aligned}
d_{i} & =\max _{x_{i} \in\left[0, e_{i}\right]} b_{i}\left(x_{i}\right), & \\
d_{12} & =\max _{x_{1} \in\left[0, e_{1}\right], x_{2} \in\left[0, e_{2}\right]}\left[b_{1}\left(x_{1}\right)+b_{2}\left(x_{2}\right)\right], & i \in N, \\
d_{i 3} & =\max _{x_{i} \in[0, e], x_{3} \in\left[0, e_{3}+e-x_{i}\right]}\left[b_{i}\left(x_{i}\right)+b_{3}\left(x_{3}\right)\right], & i=1,2, \\
d_{N} & =\max _{x_{1}, x_{2} \in[0, e], x_{3} \in\left[0, e_{3}+2 e-x_{1}-x_{2}\right]}\left[b_{1}\left(x_{1}\right)+b_{2}\left(x_{2}\right)+b_{3}\left(x_{3}\right)\right] . &
\end{aligned}
$$

In Section 2.1 it is assumed that for every agent its satiation point $x_{i}^{S}$ exceeds its own inflow $e_{i}$, and therefore $d_{i}=b_{i}\left(e_{i}\right), i \in N$. Furthermore, the $\mathrm{V}$-shaped river structure imposes that 
water flows between agents 1 and 2 are impossible, and this implies $d_{12}=b_{1}\left(e_{1}\right)+b_{2}\left(e_{2}\right)=$ $b_{1}(e)+b_{2}(e)$ because $e_{1}=e_{2}=e$. Recall also that we assumed that the marginal benefit of agent 3 in $e_{3}$ is higher than the marginal benefit of agent $i, i=1,2$ in $e$. This implies that in a solution of the maximization problem to find $d_{i 3}$ we must have, on the one hand, that $x_{3}>e_{3}$ and $x_{i}<e$. On the other hand $x_{3}<x_{3}^{S}$, because at $x_{3}=x_{3}^{S}>e_{3}$ the marginal benefit of water is zero for agent 3 , whereas that of his upstream trading partner $i$ is positive. So, agent 3 and upstream agent $i$ will trade, but never up to agent 3 's satiation point. It follows that

$$
d_{i 3}=\max _{x_{i} \in[0, e]}\left[b_{i}\left(x_{i}\right)+b_{3}\left(e_{3}+e-x_{i}\right)\right], i=1,2
$$

Furthermore, the pair of agents $i$ and 3 will be better off if agent $j, j \neq i, 3$, also joins their coalition. To see this, if agent $j$ would be excluded this agent's marginal benefit at $x_{j}=e$ would be lower than that of agent $i$ who supplies a positive amount to agent 3. By letting agent $j$ take over part of $i$-th agent's supply to agent 3 , the total benefit of all three agents can increase. So, it follows that

$$
d_{N}=\max _{x_{1}, x_{2} \in[0, e]}\left[b_{1}\left(x_{1}\right)+b_{2}\left(x_{2}\right)+b_{3}\left(e_{3}+2 e-x_{1}-x_{2}\right)\right] .
$$

For similar reasons as before, we have $x_{1}=x_{2}<e$ and $x_{3} \in\left(e_{3}, x_{3}^{S}\right)$ in the optimum. Clearly, $d_{N}>d_{i 3}$ and, by the strict concavity of the benefit functions $d_{N}<d_{13}+d_{23}$. By symmetry of agent 1 and 2 , we have $d_{1}=d_{2}$ and $d_{13}=d_{23}$. From this it follows that without loss of generality we may normalize the benefit functions in such a way that the disagreement values become

$$
d_{1}=d_{2}=d_{3}=0, d_{12}=0, d_{13}=d_{23}=1, \text { and } d_{N}=1+\varepsilon,
$$

with $\varepsilon \in(0,1)$. Obviously, the normalized disagreement values in the $\mathrm{V}$-shaped river sharing problem satisfy Assumption 3.1. This parameter $\varepsilon$ can be interpreted as a productivity parameter of water for the second of the two "units" $e$ of water that the upstream agents hold. Trading at most one unit $e$ generates an incremental benefit of 1 and due to diminishing returns the second unit $e$ generates less incremental value. The limiting cases of $\varepsilon$ are also of interest. For instance, $\varepsilon=0$ corresponds to zero incremental value for water from the second upstream agent. This is essentially a cooperative game with transferable utility called the Glove Game, where only pairs of gloves have value, agent 3 holds, say, a left-hand glove and each upstream agent holds a right-hand glove. And $\varepsilon=1$ corresponds to a linear benefit function for agent 3 or constant marginal benefits from water.

Due to the absence of externalities, the $\gamma$-Core coincides with the Core. Furthermore, it is easily verified that the cooperative game is balanced and, thus, the Core is nonempty. The Core is equal to the convex hull of the four vectors $(\varepsilon, \varepsilon, 1-\varepsilon),(0, \varepsilon, 1),(\varepsilon, 0,1)$ and 


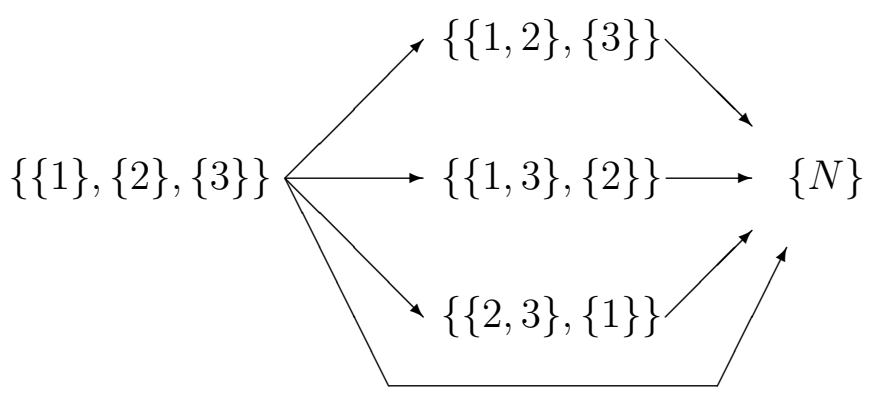

Figure 2: All coalition transitions that are possible in the $\mathrm{V}$-shaped river sharing problem.

$(0,0,1+\varepsilon)$ that support its corners, which makes it diamond shaped.

Finally, it is important to keep in mind that the normalization of disagreement values into a single productivity parameter still covers the entire class of $\mathrm{V}$-shaped river sharing problems. Given that the common discount factor $\delta \in[0,1)$ is also a parameter of the CBG $[0,1]$, we are now ready to analyze the associated $\mathrm{CBG}$ for all parameter values of $\varepsilon \in(0,1)$ and $\delta \in[0,1)$.

\subsection{Main Results}

In this section, we provide closed-form solutions for the value functions of the CBG with the (normalized) disagreement values given in Section 4.1. Because the underlying derivations are long and tedious, these are deferred to an online appendix where we also derive rather cumbersome closed-form solutions for the mixed stage-game MPE strategies. Therefore, we will discuss the main insights that are obtained from the equilibrium analysis without going into too much technical detail.

To begin with, we apply the results obtained in Section 3.2 to the $\mathrm{V}$-shaped river sharing problem. The existence of MPE follows immediately from the general CBG. As mentioned in Section 4.1, the disagreement values satisfy Assumption 3.1 for all $\varepsilon \in(0,1)$. So, the next proposition follows immediately form Lemma 3.2 .

Proposition 4.1. For a $V$-shaped river sharing problem with $\varepsilon \in(0,1)$, in every mixed $M P E$ of the CBG it holds that, for every coalition structure $\pi \neq \pi^{N}$, every proposing coalition $C \in \pi$ proposes to merge with at least one other coalition and all responders accept.

As explained in Section 3.2, even though the proposer may randomize over several proposals, all these proposals will be accepted and some agreement will be reached for sure. This implies that the grand coalition of all three agents forms in at most two rounds. Figure 2 shows the four possible transitions from the initial coalitional structure $\pi^{0}$ to the grand coalition $\pi^{N}$. There are three transitions out of the initial state that involve two bargaining rounds and in which first a coalition with two agents forms before the grand coalition forms. In the fourth transition the grand coalition forms immediately in a single transition. 
The logic of subgame perfectness that underlies the MPE concept dictates that we first solve for the relevant subgames. As already mentioned, all subgames associated with coalition structure $\pi^{N}$, the absorbing state, are trivial and have $v_{N}\left(\pi^{N}\right)=1+\varepsilon$. Next, we need to consider subgames associated with a coalition structure $\pi^{i}=\{\{i\}, N \backslash\{i\}\}, i \in N$, in which player $i$ is still single and the other two players have formed a coalition. In what follows, we simply write $i$ for $\{i\}$ and $i j$ for $\{\{i\},\{j\}\}$. Because $\bar{\delta}\left(\pi^{i}\right)=1$, according to the results in Section 3.2, we have immediate agreement in any subgame associated with the state $\pi^{i}$. Application of Lemma 3.3 yields the following result.

Proposition 4.2. For the $V$-shaped river sharing problem given by (8), we have for $i, j=$ $1,2, i \neq j$, that

$$
\begin{array}{ll}
v_{j 3}\left(\pi^{i}\right)=1+\frac{2}{3} \varepsilon, & v_{i}\left(\pi^{i}\right)=\frac{1}{3} \varepsilon \\
v_{12}\left(\pi^{3}\right)=\frac{2}{3}(1+\varepsilon), & v_{3}\left(\pi^{3}\right)=\frac{1}{3}(1+\varepsilon) .
\end{array}
$$

The MPE strategies underlying this result are pure strategies. This result implies that we have exhausted all subgames that might be reached from the initial coalition structure.

Following Gomes (2005), we consider net surpluses instead of the value $v_{i}\left(S, t\left(\pi^{0}\right) \mid \pi^{0}\right)$ of proposing $\left(S, t\left(\pi^{0}\right)\right)$. Notice that the net surplus for a pair $S=i j$ is equal to

$$
v_{i}\left(i j, t\left(\pi^{0}\right) \mid \pi^{0}\right)-t_{i}\left(\pi^{0}\right)=v_{j}\left(i j, t\left(\pi^{0}\right) \mid \pi^{0}\right)-t_{j}\left(\pi^{0}\right),
$$

which we denote as $a$ for $i j=12$ and $b$ for $i j=13,23$ by symmetry. Similarly, for $S=N$ instead of $S=\{N\}$,

$$
v_{1}\left(N, t\left(\pi^{0}\right) \mid \pi^{0}\right)-t_{1}\left(\pi^{0}\right)=v_{2}\left(N, t\left(\pi^{0}\right) \mid \pi^{0}\right)-t_{2}\left(\pi^{0}\right)=v_{3}\left(N, t\left(\pi^{0}\right) \mid \pi^{0}\right)-t_{3}\left(\pi^{0}\right),
$$

which we denote as $c$.

Now we are ready to consider the initial coalition structure $\pi^{0}$. At this coalition structure, every proposer $i$ randomizes over proposals $\left(S, t\left(\pi^{0}\right)\right)$ that maximize their net surplus, because for every player $i \in N$ it holds that

$$
\max _{S \subseteq \pi, S \ni i}\left[v_{i}\left((S, t(\pi)) \mid \pi^{0}\right)-t_{i}\left(\pi^{0}\right)\right]=v_{i}^{*}\left(\pi^{0}\right)-t_{i}\left(\pi^{0}\right)
$$

where the last expression also appears as the first term on the right-hand side of (5). To further simplify notation, we write $v_{3}\left(\pi^{0}\right)=v_{3}$ and, by symmetry of agent 1 and $2, v_{1}\left(\pi^{0}\right)=$ 
$v_{2}\left(\pi^{0}\right)=v$. Then, after substituting (8) and (1) in (9) we obtain

$$
\begin{aligned}
a & =\delta v_{12}\left(\pi^{3}\right)+(1-\delta) d_{12}\left(\pi^{3}\right)-2 \delta v=\frac{2(1+\varepsilon) \delta}{3}-2 \delta v, \\
b & =\delta v_{13}\left(\pi^{2}\right)+(1-\delta) d_{13}\left(\pi^{2}\right)-\delta v-\delta v_{3}=\delta v_{23}\left(\pi^{1}\right)+(1-\delta) d_{23}\left(\pi^{1}\right)-\delta v-\delta v_{3} \\
& =\frac{3+2 \varepsilon}{3} \delta+(1-\delta)-\delta v-\delta v_{3}, \\
c & =d_{N}(\{N\})-2 \delta v-\delta v_{3}=1+\varepsilon-2 \delta v-\delta v_{3} .
\end{aligned}
$$

So, agent 1 proposes to form ${ }^{13}\{1,2\}$ if $a>\max [b, c]$, to form $\{1,3\}$ if $b>\max [a, c]$ and to form the grand coalition $N$ if $c>\max [a, b]$. In case of equalities the proposer randomizes, so 1 randomizes over forming $\{1,2\}$ or $\{1,3\}$ if $a=b$. Similar for the agents 2 and 3 . Notice that agent 3 always randomizes over $\{1,3\}$ and $\{2,3\}$ if $b>c$, and randomizes over $\{1,3\}$, $\{2,3\}$ and $N$ if $b=c$.

The values of $a, b$ and $c$ and so the proposals in the first round of the bargaining process depend on the parameters $\delta$ and $\varepsilon$. It appears that the $(\delta, \varepsilon)$ space can be divided in five different regions, denoted $A, B, C, D$ and $E$. Each region corresponds to a specific ordering of the three values $a, b$ and $c$. The regions are given in Table 1 , together with the ordering of $a, b$ and $c$ and the proposals in the corresponding equilibrium. Notice that not all orderings occur, for instance there are no values of $\delta$ and $\varepsilon$ such that $a>\max [b, c]$. Consequently, there is no equilibrium in which the agents 1 and 2 propose to form coalition $\{1,2\}$ with probability 1 . But it might happen that $a=b=c$, in that case every agent mixes over all three possible proposals, so in this case the proposal to form $\{1,2\}$ might occur with positive probability. Note that in Region $B$ agent 3 proposes to form $\{1,3\}$ and $\{2,3\}$ with equal probabilities $\frac{1}{2}$, since agents 1 and 2 are symmetric.

The five regions dividing the $[0,1] \times[0,1] \in R^{2}$ space of all pairs $(\delta, \varepsilon)$ are shown in Figure 3. Because region $E$ is very small, Figure 4 zooms in around this region. From the figures we make the following observations.

1. For Region $A$ in which everyone proposes the grand coalition, we have $\delta \leq \frac{3 \varepsilon}{1+3 \varepsilon}$ by applying Theorem 3.4. The intuition is that if $\delta$ is sufficiently small, the loss due to the delay of forming the efficient grand coalition is large for the proposing agent and for the responding agents the present value of $v_{i}\left(\pi^{0}\right)$ is lowered, hence all agents have incentives to propose the grand coalition immediately.

2. For a fixed $\varepsilon$, proposing to form a partial coalition instead of $N$ becomes more plausible when $\delta$ increases. For instance, in regions $B$ and $D$, every agent proposes a partial coalition. An intuitive explanation is that when $\delta$ is sufficiently large, every agent is searching for a better bargaining position in order to extract future surplus. It seems counter-intuitive that agents 1 and 2 propose the coalition $\{1,2\}$ in $\pi^{0}$ which does

\footnotetext{
${ }^{13}$ Formally the proposal should be $\{\{1\},\{2\}\}$. This abbreviation follows hereafter.
} 
not generate any surplus in current round since $d_{12}\left(\pi^{3}\right)=0$. However, in the next bargaining round, coalition 1 and 2 can act as a monopolist and extract a larger share of the surplus when forming the grand coalition with agent 3 .

3. $(\delta, \varepsilon) \approx(0.875,0.95)$ serves as an important threshold point for different equilibria. We observe the equilibria of $A, C, B$ and $D$ for $\varepsilon$ below 0.95 while above that, we observe equilibria of $A, C, E$ and $D$. 


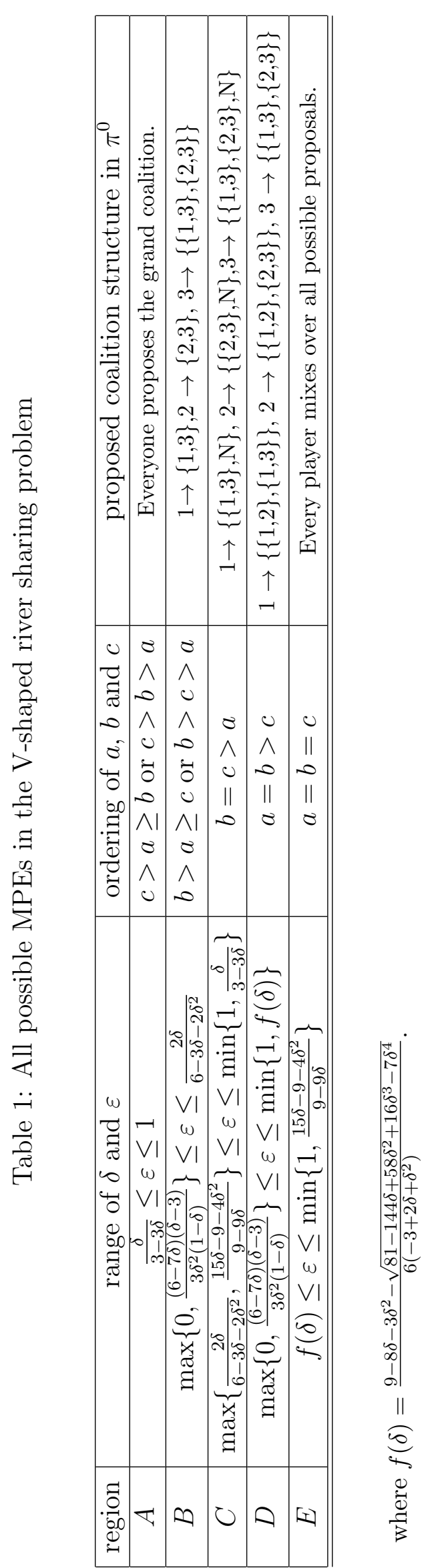




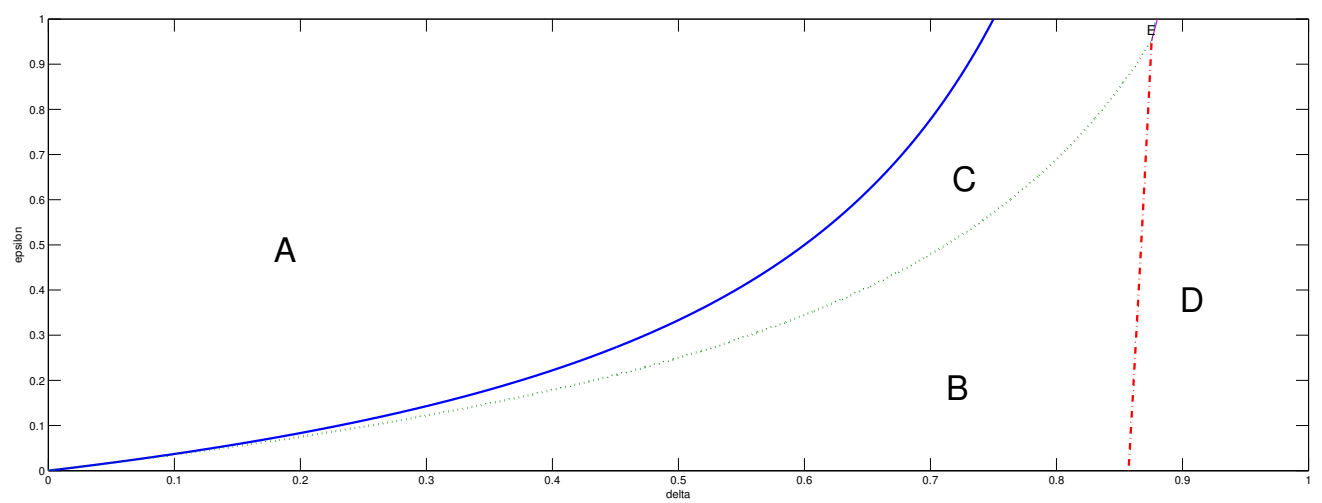

Figure 3: The regions of different equilibria in the $(\delta, \varepsilon)$ space

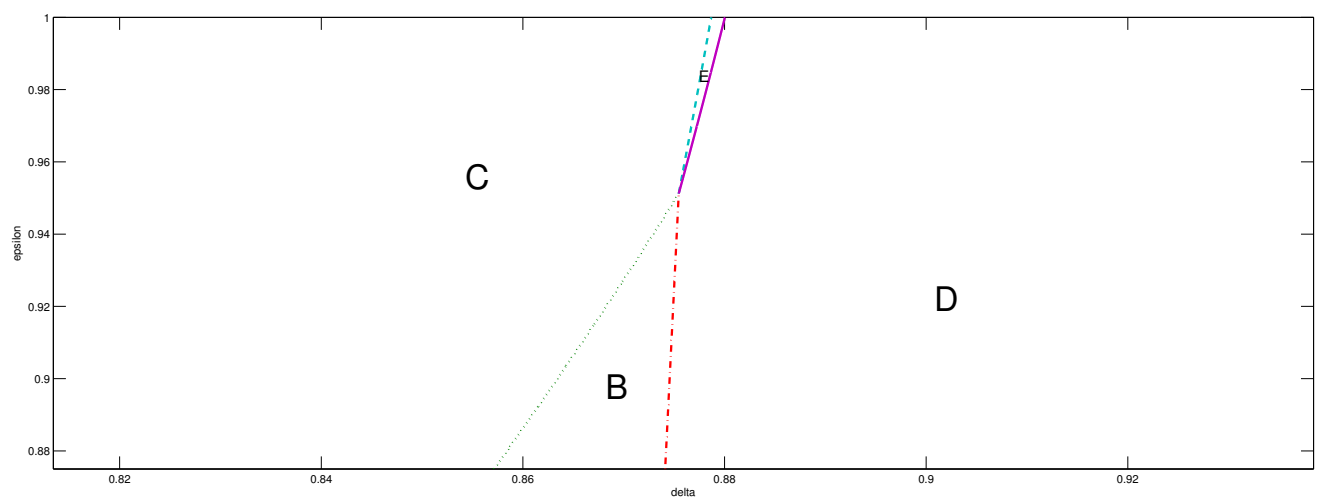

Figure 4: Region $E$ in the $(\delta, \varepsilon)$ space

Having found the optimal proposals of the agents in $\pi^{0}$, for each region we can calculate for each agent $i \in N$ the expected payoff of the optimal proposal $v_{i}^{*}\left(\pi^{0}\right)$ by Equation (5) and then derive the values $v_{i}\left(\pi^{0}\right)$, shortly denoted by $v_{i}\left(\pi^{0}\right)$. Proposition 4.3 summarizes for each of the five regions the optimal proposals of the agents and the corresponding values $v_{i}\left(\pi^{0}\right)$.

Proposition 4.3. In a MPE of the $C B G$ for the $V$-shaped river sharing problem, the proposals in the initial coalitional structure $\pi^{0}$ are randomized over the coalitions depending on 
$(\delta, \varepsilon)$ according to Table 1 . Moreover the values $v_{i}\left(\pi^{0}\right), i \in N$, are given by

$$
\begin{aligned}
A: v_{1}\left(\pi^{0}\right) & =v_{2}\left(\pi^{0}\right)=v_{3}\left(\pi^{0}\right)=\frac{1+\varepsilon}{3} ; \\
B: v_{1}\left(\pi^{0}\right) & =v_{2}\left(\pi^{0}\right)=\frac{2(1-\delta)(2 \delta \varepsilon+3)+\delta \varepsilon(3-2 \delta)}{3(6-5 \delta)} \\
v_{3}\left(\pi^{0}\right) & =\frac{(2-\delta)(2 \delta \varepsilon+3)-\delta^{2} \varepsilon}{3(6-5 \delta)} ; \\
C: v_{1}\left(\pi^{0}\right) & =v_{2}\left(\pi^{0}\right)=\frac{3-2 \delta}{3 \delta} \varepsilon, v_{3}\left(\pi^{0}\right)=\frac{3(1-\varepsilon)+4 \varepsilon \delta}{3(3-2 \delta)} ; \\
D: v_{1}\left(\pi^{0}\right) & =v_{2}\left(\pi^{0}\right)=\frac{4(1+\varepsilon) \delta+\delta \varepsilon(g(\delta, \varepsilon)+1)}{18-3 \delta(1-g(\delta, \varepsilon))} \\
v_{3}\left(\pi^{0}\right) & =\frac{2(1+\varepsilon) \delta-6 \delta v_{1}\left(\pi^{0}\right)+\delta(1+\varepsilon)(2-g(\delta, \varepsilon))}{9-3 \delta-3 \delta g(\delta, \varepsilon)} \\
E: v_{1}\left(\pi^{0}\right) & =v_{2}\left(\pi^{0}\right)=\frac{3-2 \delta}{3 \delta} \varepsilon, v_{3}\left(\pi^{0}\right)=\frac{(1+\varepsilon)(3-2 \delta)}{3 \delta} .
\end{aligned}
$$

where:

$$
g(\delta, \varepsilon)=\frac{1}{-2 \delta(1-\delta)}\left(3-4 \delta+3 \delta^{2}-3 \delta \varepsilon+3 \delta^{2} \varepsilon-\sqrt{\Delta}\right)
$$

and

$$
\Delta=81-180 \delta+130 \delta^{2}-28 \delta^{3}+\delta^{4}-18 \delta \varepsilon+30 \delta^{2} \varepsilon-18 \delta^{3} \varepsilon+6 \delta^{4} \varepsilon+9 \delta^{2} \varepsilon^{2}-18 \delta^{3} \varepsilon^{2}+9 \delta^{4} \varepsilon^{2} .
$$

In regions $B, C, D$ and $E$, there is an efficiency loss due to the delay of the formation of the grand coalition. This efficiency loss depends on the probability that the grand coalition is proposed by the agents. For instance, the efficiency loss for Region $B$ is $(1-\delta) \varepsilon$, which is exactly the loss due to the delay of the formation of the grand coalition for one round.

Consider a horizontal line in Figure 3 that lies below Region $E$, which means fix $\varepsilon \in$ $(0,0.95)$. Starting with $\delta$ close enough to 0 , we are in Region $A$ where all agents propose the grand coalition and all responding agents immediately agree. All results for Region $A$ follow by applying the results derived in Section 4.2. By letting $\delta$ increase, we move into Region $C$, where then upstream agents randomize between proposing the grand coalition and a bilateral agreement with the downstream agent, and the downstream agent also randomizes between the bilateral agreement with the upstream agent and the grand coalition. So, there still is a positive probability of forming the grand coalition immediately but also a positive probability of gradual coalition formation. If $\delta$ increases further, we reach Region $B$ where each of the upstream agents proposes a bilateral agreement with the downstream agent and the downstream agent randomizes the two possible bilateral agreements with the upstream agents. In this region, gradual coalition formation will occur for sure, because none of the agents proposes the grand coalition. If $\delta$ is sufficiently close to 1 , we reach Region $D$. In this region, the upstream agents randomize between forming a monopoly and a bilateral agreement with the downstream agent, who follows a similar randomization as in Region 
$B$. This implies that gradual coalition formation will also occur for sure in Region $D$. For $\varepsilon \in(0.95,1)$, the horizontal line also crosses Region $E$. In this region, every agent randomizes over all three possible coalitions and every sequence of gradual coalition formation can occur with positive probability.

The vector of values for Region $A$ lies outside the Core if $v_{1}\left(\pi^{0}\right)>\varepsilon$, which implies $\varepsilon>\frac{1}{2}$. So, the MPE in the CBG does not necessarily yield solutions in the Core. The efficiency loss in the other regions makes the comparison to the Core meaningless. However, Region $D$ is the relevant region for sufficiently large discount factors and, by taking the limit $\delta$ goes to 1 , the inefficiency of the MPE vanishes in the limit. Taking the limit yields $\lim _{\delta \rightarrow 1} g(\delta, \varepsilon)=1$ and, hence, we obtain the efficient limit outcome

$$
\lim _{\delta \rightarrow 1} v_{1}\left(\pi^{0}\right)=\lim _{\delta \rightarrow 1} v_{2}\left(\pi^{0}\right)=\frac{1}{3} \varepsilon+\frac{2}{9}, \quad \lim _{\delta \rightarrow 1} v_{3}\left(\pi^{0}\right)=\frac{1}{3} \varepsilon+\frac{5}{9}
$$

Agent 3 receives at most his worst outcome $1-\varepsilon$ in the Core if and only if $\varepsilon \geq \frac{1}{3}$. So, we conclude that for $\varepsilon \geq \frac{1}{3}$ the vector of limit outcomes lies in the Core. This also implies that this vector lies outside the Core otherwise.

\section{Concluding Remarks}

In this paper, we identified gradual coalition formation and the strategic timing of becoming a member of an IEA as an important and relevant research topic. We applied the Coalitional Bargaining Game (CBG) in Gomes (2005) to deal with these issues. Our results are twofold: extending general results and an illustration of the merits of applying the CBG. For the general CBG we derive the necessary and sufficient condition that entails immediate agreement on the grand coalition in every possible coalition structure in the CBG. This condition states that the common discount factor, which describes the agents' time preferences, should be smaller than some upper bound. This result extends a result in Gomes (2005). This condition for immediate agreement on the grand coalition is of interest, because this enables us to identify which real-life environmental problems will be easy to deal with through swift negotiations by the stakeholders involved.

One area where there is a need for IEAs are international rivers. Therefore, we also applied the CBG to a specific simple river sharing problem with a V-shaped river structure with two symmetric upstream agents and one downstream agent. Although simple in nature, this problem has several intriguing features as argued in the introduction. We normalized this problem in such a way that we obtained a single productivity parameter describing the class of $\mathrm{V}$-shaped river sharing problems under consideration. Together with the common discount factor of the CBG we have two parameters in our application. Nevertheless, we obtain a rich spectrum of equilibrium behavior. In two large regions and for sufficiently patient agents, gradual coalition formation occurs for sure. In one region, both upstream 
agents propose with positive probability to form a monopoly first, before bargaining with the downstream agent. These results provide valuable insights how coalition formation emerges over time. On the one hand, it seems counter-intuitive that two upstream agents that cannot trade water with each other form a coalition knowing they do not receive extra benefit in the short run. On the other hand, those two upstream agents will act as a monopolist and extract surplus in the future negotiations. By acting in this way, forming the grand coalition will be delayed.

\section{References}

Ambec, S. and L. Ehlers (2008). Sharing a river among satiable agents. Games and Economic Behavior 64, 35-50.

Ambec, S. and Y. Sprumont (2002). Sharing a river. Journal of Economic Theory 10\%, 453-462.

Carraro, C., J. Eyckmans, and M. Finus (2006). Optimal transfers and participation decisions in international environmental agreements. Review of International Organizations 1, 379-396.

Carraro, C., C. Marchiori, and A. Sgobbi (2005a). Advances in negotiation theory: bargaining, coalitions, and fairness. World Bank Policy Research Working Paper 3642, WPS3641, The World Bank, Washington D.C., http://econ.worldbank.org.

Carraro, C., C. Marchiori, and A. Sgobbi (2005b). Applications of negotiation theory to water issues. World Bank Policy Research Working Paper 3641, WPS3641, The World Bank, Washington D.C., http://econ.worldbank.org.

Chander, P. and H. Tulkens (1995). A Core-theoretical solution for the design of cooperative agreements on transfrontier pollution. International Tax and Public Finance 2, 279-293.

Chander, P. and H. Tulkens (1997). The Core of an economy with multilateral environmental externalities. International Journal of Game Theory 26, 379-401.

Chatterjee, K., B. Dutta, D. Ray, and K. Sengupta (1993). A noncooperative theory of coalitional bargaining. Review of Economic Studies 60, 463-477.

Eyckmans, J. and M. Finus (2004). An almost ideal sharing scheme for coalitional games with externalities. Working Paper, Katholieke Universiteit Leuven.

Germain, M., P. Toint, H. Tulkens, and A. de Zeeuw (2003). Transfers to sustain dynamic core-theoretic cooperation in international stock pollutant games. Journal of Economic Dynamics and Control 28, 79-99.

Gomes, A. (2005). Multilateral contracting with externalities. Econometrica 73, 1329 1350. 
Gomes, A. and P. Jehiel (2005). Dynamic processes of social and economic interactions: On the persistence of inefficiencies. Journal of Political Economy 113, 626-667.

Houba, H., K. Sneek, and F. Várdy (2000). Can negotiations prevent fish war? Journal of Economic Dynamics and Control 24, 1265-1280.

Houba, H., G. van der Laan, and Y. Zeng (2013). Asymmetric Nash solutions in the river sharing problem. Working Paper, Tinbergen Institute.

Macho-Stadler, I., D. Pérez-Castrillo, and D. Wettstein (2007). Sharing the surplus: an extension of the Shapley value for environments with externalities. Journal of Economic Theory 135, 339-356.

McGinty, M., G. Milam, and A. Gelves (2012). Coalition stability in public goods provision: Testing a new allocation rule. Environmental and Resource Economics 52, $327-345$.

Muthoo, A. (1999). Bargaining theory with applications. Cambridge: Cambridge University Press.

Nagashima, M., R. Dellink, E. van Ierland, and H.-P. Weikard (2009). Stability of international climate coalitions - a comparison of transfer schemes. Ecological Economics 68, $1476-1487$.

Okada, A. (1996). A noncooperative coalitional bargaining game with random proposers. Games and Economic Behavior 16, 97-108.

Okada, A. (2000). The efficiency principle in non-cooperative coalitional bargaining. Japanese Economic Review 51, 34-50.

Okada, A. (2011). Coalitional bargaining games with random proposers: Theory and application. Games and Economic Behavior 73, 227-235.

van den Brink, R., G. van der Laan, and N. Moes (2012). Fair agreements for sharing international rivers with multiple springs and externalities. Journal of Environmental Economics and Management 63, 388-403.

Weikard, H.-P. (2009). Cartel stability under an optimal sharing rule. The Manchester School 77\%, 575-593.

Weikard, H.-P., M. Finus, and J.-C. Altamirano-Cabrera (2006). The impact of surplus sharing on the stability of international climate agreements. Oxford Economic Papers 58, 209-232. 


\section{Appendix}

\section{Proof of Lemma 3.2}

Consider the proposal $(S, t(\pi))=(\pi, t(\pi))$ for coalition $C$, then in (5) the first term on the right-hand side becomes

$$
\begin{aligned}
& (1-\delta) d_{N}(\{N\})+\delta v_{N}(\{N\})-\sum_{B \in \pi}\left[(1-\delta) d_{B}(\pi)+\delta v_{B}(\pi)\right] \\
= & (1-\delta)\left[d_{N}(\{N\})-\sum_{B \in \pi} d_{B}(\pi)\right]+\delta\left[v_{N}(\{N\})-\sum_{B \in \pi} v_{B}(\pi)\right]>0,
\end{aligned}
$$

because the first term is positive by Assumption 3.1 and the second term is nonnegative. So, the maximum has to be positive, which rules out that proposing $(S, t(\pi))=(\{C\}, t(\pi))$ is optimal. QED.

\section{Proof of Lemma 3.3}

If $\sigma_{B}(\pi \mid \pi)=1$ is optimal for every $B \in \pi, \pi \neq\{N\}$, then $\sum_{\substack{S \subseteq B \\ S \subsetneq C}} \sigma_{B}(S \mid \pi)=1$ and $\sum_{\substack{S \subset B, C \notin S \\ S \ni B}} \sigma_{B}(S \mid \pi)=0$. So, Equation (5) degenerates to

$$
\begin{aligned}
v_{C}(\pi)= & p_{C}\left[(1-\delta) d_{N}(\{N\})+\delta v_{N}(\{N\})-\sum_{B \in \pi}\left[(1-\delta) d_{B}(\pi)+\delta v_{B}(\pi)\right]\right] \\
& +\left[(1-\delta) d_{C}(\pi)+\delta v_{C}(\pi)\right] .
\end{aligned}
$$

Summing over all $B \in \pi$ and substituting $v_{N}(\{N\})=d_{N}(\{N\})$ yields $\sum_{B \in \pi} v_{B}(\pi)=$ $d_{N}(\{N\})$. Then the equation for $v_{C}(\pi)$ can be rewritten into

$$
(1-\delta) v_{C}(\pi)=(1-\delta) d_{C}(\pi)+p_{C}\left[(1-\delta)\left(d_{N}(\{N\})-\sum_{B \in \pi} d_{B}(\pi)\right)+\delta\left[d_{N}(\{N\})-\sum_{B \in \pi} v_{B}(\pi)\right]\right]
$$

and we immediately obtain

$$
v_{C}(\pi)=d_{C}(\pi)+p_{C}\left(d_{N}(\{N\})-\sum_{B \in \pi} d_{B}(\pi)\right)
$$

QED. 


\section{Proof of Theorem 3.4}

Only if: The equilibrium conditions such that every proposer in the subgame at state $\pi$ proposes the grand coalition impose that

$$
\begin{aligned}
& (1-\delta) d_{N}(\{N\})+\delta v_{N}(\{N\})-\sum_{B \in \pi}\left[(1-\delta) d_{B}(\pi)+\delta v_{B}(\pi)\right] \\
\geq & (1-\delta) d_{S} \cup\left(\pi_{S}\right)+\delta v_{S} \cup\left(\pi_{S}\right)-\sum_{B \in S}\left[(1-\delta) d_{B}(\pi)+\delta v_{B}(\pi)\right],
\end{aligned}
$$

for all $S \subset \pi$. Rewriting several times yields

$\delta\left[v_{S} \cup\left(\pi_{S}\right)-d_{S \cup}\left(\pi_{S}\right)+\left(d_{N}(\{N\})-\sum_{B \in \pi} d_{B}(\pi)\right) \sum_{B \in \pi \backslash S} p_{B}\right] \leq d_{N}(\{N\})-d_{S \cup}\left(\pi_{S}\right)-\sum_{B \in \pi \backslash S} d_{B}(\pi)$.

Notice that we consider strategies in which every proposer in every subgame $\pi^{\prime} \leq{ }_{p} \pi$ proposes the grand coalition. Then according to Lemma 3.3 we have

$$
v_{S \cup}\left(\pi_{S}\right)=d_{S \cup}\left(\pi_{S}\right)+p_{S} \cup\left(d_{N}(\{N\})-\sum_{B \in \pi_{S}} d_{B}\left(\pi_{S}\right)\right) .
$$

By further substitution, we get

$$
\begin{aligned}
& \delta\left[p_{S \cup}\left(d_{N}(\{N\})-\sum_{B \in \pi_{S}} d_{B}\left(\pi_{S}\right)\right)+\left(d_{N}(\{N\})-\sum_{B \in \pi} d_{B}(\pi)\right) \sum_{B \in \pi \backslash S} p_{B}\right] \\
& \leq d_{N}(\{N\})-d_{S} \cup\left(\pi_{S}\right)-\sum_{B \in \pi \backslash S} d_{B}(\pi) .
\end{aligned}
$$

Since the grand coalition is efficient, the term in the square bracket on the left-hand side is positive. Then, $\delta \leq \delta_{\pi}(S)$ follows immediately. By taking the minimum over all $S^{\cup}$ we obtain $\delta \leq \delta(\pi)$ for every coalition structure $\pi$. Finally, by taking the minimum over $\pi$ and coalitions structures coarser than $\pi$, i.e., $\left\{\pi^{\prime} \leq_{p} \pi:\left|\pi^{\prime}\right| \neq 1\right\}$, we get the desired result.

If: When $\delta \leq \bar{\delta}(\pi)$, we prove the result recursively. Let $\pi$ be a coalition structure with $|\pi|=2$, so $\pi$ consists of two coalitions, say $C_{1}$ and $C_{2}$. Then

$$
\bar{\delta}(\pi)=\min _{\left\{\pi^{\prime} \leq p \pi:\left|\pi^{\prime}\right| \neq 1\right\}} \delta\left(\pi^{\prime}\right)=\delta(\pi)=\min _{S=\left\{C_{1}\right\}, S=\left\{C_{2}\right\}} \delta_{\pi}(S)
$$

Taking either $S=\left\{C_{1}\right\}$ or $S=\left\{C_{2}\right\}$ yields $\delta_{\pi}(S)=1$, implying that from a coalition structure with two coalitions the grand coalition is formed immediately for every value of $\delta$. Now consider $|\pi|=3$, when $\delta \leq \bar{\delta}(\pi)$, it can be easily shown that for every proposer in $\pi$, it is optimal to propose the grand coalition immediately. For $\pi=\pi^{0}$ and $|\pi|>3$, we can deduce backwards in a similar way. QED. 